\title{
PICO PRÉ-OVULATÓRIO DE LH EM FÊMEAS NELORE (BOS TAURUS INDICUS) SINCRONIZADAS*
}

\author{
LH PRE-OVULATORY PEAK IN SYNCHRONIZED NELLORE BREED \\ (BOS TAURUS INDICUS) FEMALES
}

\author{
Ferraz, H.T. ${ }^{1}$, B.D. Oliveira Filho ${ }^{2}$, M.L. Gambarini' ${ }^{2}$, M.A.O. Viu ${ }^{1}$, D.T. Lopes ${ }^{3}$ \\ e A.P.F. Sousa ${ }^{4}$
}

\begin{abstract}
${ }^{1}$ Escola de Veterinária. Universidade Federal de Goiás. Câmpus Jataí. Brasil. htferraz@gmail.com ${ }^{2}$ Escola de Veterinária. Universidade Federal de Goiás. Câmpus Goiânia. Brasil. ${ }^{3}$ Escola de Veterinária. Universidade Federal de Goiás. Brasil. ${ }^{4}$ Médica Veterinária. Autônoma.
\end{abstract}

\section{PalaVRas ChaVe ADICIONAIS}

Benzoato de estradiol. Bovinos. IATF. Progestágenos. Reprodução.

\section{RESUMO}

Desenvolveu-se este trabalho com o objetivo de avaliar a freqüência de pulsos e a amplitude do pico pré-ovulatório do hormônio luteinizante (LH) de novilhas e vacas Nelore submetidas a um protocolo hormonal. Em seis fêmeas de cada categoria utilizou-se o seguinte protocolo: colocação do implante de norgestomet (NOR) e aplicação de 2,0 mg de benzoato de estradiol (BE) no dia zero, com retirada após oito dias, seguida da aplicação de $0,5 \mathrm{mg}$ de cloprostenol sódico. Após 24 horas (dia nove) $1,0 \mathrm{mg}$ de BE. As colheitas de sangue iniciaram-se 27,00 horas após a aplicação da segunda dose de BE, sendo repetidas a cada 15 minutos por um período de seis horas. A determinação dos níveis séricos de LH foi feita pelo método de radioimunoensaio. Em uma novilha e em quatro vacas pôde-se observar o pico pré-ovulatório de LH. Na novilha o pico foi verificado na primeira colheita, 27,00 horas após a indução da ovulação. Nas vacas este ocorreu, em média, 28,20 $\pm 0,55$ horas após a aplicação do $B E$. A amplitude máxima verificada foi, em média, de $4,58 \pm 0,67 \mathrm{ng} / \mathrm{ml}$. Nos animais em que não se observou o pico de LH, as concentrações médias foram de $0,90 \pm 0,03 \mathrm{ng} / \mathrm{ml}$ para as novilhas e de $0,86 \pm 0,03 \mathrm{ng} / \mathrm{ml}$ para as vacas. Sugere-se que

*Parte da Dissertação de Mestrado do primeiro autor.

Recibido: 13-3-08. Aceptado: 16-6-08.

\section{ADDITIONAL KEYWORDS}

Estradiol benzoate. Bovine. TAI. Progestins. Reproduction.

nas novilhas o pico pré-ovulatório de LH, provavelmente, tenha ocorrido mais precocemente que nas vacas. No entanto, são necessários mais estudos sobre o momento do pico de LH em fêmeas bovinas com ovulação sincronizada.

\section{SUMMARY}

The aim of this work was to evaluate the frequency of pulses and the amplitude of the preovulatory peak of the luteinizing hormone (LH) in Nellore heifers and cows submitted to a hormonal protocol. In six females owning to each category the following protocol was used: placement of the norgestomet implant (NOR) and application of 2.0 $\mathrm{mg}$ of estradiol benzoate (EB) on day zero, with retreat after eight days, followed by the application of $0.5 \mathrm{mg}$ of sodic cloprostenol. After 24 hours (day nine) $1.0 \mathrm{mg}$ of EB was used. The blood sampling was initiated 27.00 hours after the application of the second dose of EB, and repeated every 15 minutes for a six hours period. Seric levels of LH were detected by radioimmunoassay method. In one heifer and in four cows the preovulatory peak of LH was observed. In the heifer the peak was verified in the first sampling, 27.00 hours after the ovulation induction. In the cows this occurred on $28.20 \pm 0.55$ hours after application of EB. The maximum amplitude verified was

Arch. Zootec. 58 (224): 757-760. 2009. 
$4.58 \pm 0.67 \mathrm{ng} / \mathrm{ml}$. In the animals in which LH peak was not observed, the average concentrations were $0.90 \pm 0.03 \mathrm{ng} / \mathrm{ml}$ for the heifers and $0.86 \pm 0.03$ $\mathrm{ng} / \mathrm{ml}$ for the cows. It suggests that in the heifers the pre-ovulatory peak of LH, probably, have occurred more precociously than in the cows. However, more studies about the LH peak moment in bovine females with synchronized ovulation are needed.

\section{INTRODUÇÃO}

O Centro-Oeste brasileiro possui um rebanho bovino de aproximadamente 72 milhões de cabeças, com mais de $80 \%$ de sangue zebuíno, grande parte das quais formadoras de plantéis de cria. Por isso, dados mais precisos sobre a fisiologia reprodutiva de fêmeas Nelore submetidas a protocolos hormonais para sincronização da ovulação e realização da inseminação artificial em tempo fixo (IATF), poderiam auxiliar na determinação do melhor momento para a realização da inseminação, aumentando a eficiência destes protocolos e o retorno econômico para o produtor. Assim, desenvolveu-se este trabalho com o objetivo de avaliar a freqüência de pulsos e a amplitude do pico pré-ovulatório de hormônio luteinizante (LH) de novilhas e vacas Nelore submetidas a um protocolo hormonal para realização da IATF.

\section{MATERIAL E MÉTODOS}

Este estudo foi desenvolvido na Fazenda Santa Rosa, localizada no município de Caçu, sudoeste do Estado de Goiás, durante o mês de abril de 2006. Foram selecionadas seis novilhas com peso mínimo de $300 \mathrm{~kg}$ e idade entre 24 e 30 meses, e seis vacas entre a terceira e a quinta ordem de parição com, pelo menos, 60 dias pós-parto. Durante o período experimental as fêmeas foram mantidas em pastagem de Brachiaria brizantha cv. Marandú, próximas ao curral de manejo. Todos os animais foram submetidos a um protocolo hormonal com o uso de implantes auriculares de norgestomet. Este constou da aplicação, intra-muscular (IM), de 2,0 mg de benzoato de estradiol (BE) no momento da implantação do progestágeno, sendo este considerado o dia zero (D0) do protocolo. Após oito dias (D8), juntamente com a retirada do implante, realizou-se a aplicação, IM, de 0,50 mg de cloprostenol sódico. Depois de mais 24 horas (D9) aplicouse, IM, 1,0 mg de BE. As colheitas de sangue iniciaram-se 27,00 horas após a aplicação da segunda dose de BE, sendo repetidas a cada 15 minutos por um período de seis horas, ou seja, entre a $27^{\mathrm{a}}$ e a $33^{\mathrm{a}}$ hora após a aplicação do BE, totalizando assim 24 observações. Os animais foram mantidos nos troncos de contenção e o sangue colhido por punção da veia jugular, com auxílio de agulhas descartáveis 40x12 mm. O material obtido foi armazenado em tubos de ensaio identificados e mantidos na posição inclinada no interior de uma caixa térmica com gelo. Posteriormente procedeu-se a centrifugação a 1520 g por 15 minutos, sendo o soro obtido armazenado a $-20^{\circ} \mathrm{C}$. As amostras congeladas do soro sangüíneo foram remetidas ao Laboratório de Endocrinologia da Escola de Veterinária da UNESP - Campus de Araçatuba-SP - para a determinação dos níveis séricos de $\mathrm{LH}$ pelo método de radioimunoensaio. Os resultados obtidos foram tabulados em planilhas eletrônicas, procedendo-se então análise descritiva utilizando-se o procedimento FREQ do pacote computacional SAS (2000).

\section{RESULTADOSEDISCUSSÃO}

Em apenas uma das seis novilhas e em quatro das seis vacas, pôde-se observar o pico pré-ovulatório de LH nas amostras de sangue colhidas entre a $27^{\mathrm{a}}$ e a $33^{\mathrm{a}}$ hora após a segunda dose de BE (figura 1). Na novilha o pico foi verificado logo na primeira colheita, ou seja, 27,00 horas após a indução da ovulação. Já para as vacas este ocorreu, em média, 28,20 0 0,55 horas após a aplicação do BE. Maio et al. (2006), trabalhando com novilhas Nelore sincronizadas com o mesmo 


\section{PICO PRÉ-OVULATÓRIO DE LH EM FÊMEAS NELORE SINCRONIZADAS}

protocolo utilizado neste experimento, encontraram intervalo médio de 22,40 horas entre a aplicação do BE e o pico préovulatório de LH, com variação de 18 a 25 horas. Utilizando protocolo hormonal semelhante, Lammoglia et al. (1998) verificaram intervalo de 20 horas entre a aplicação de $0,75 \mathrm{mg}$ de BE e o pico de LH em novilhas Bos taurus taurus e de 24 horas entre a aplicação de 1,0 mg de BE e o pico deste hormônio em vacas do mesmo grupamento genético. A grande variação no momento do pico de LH após o uso do BE e o fato deste ocorrer, provavelmente, mais precocemente em novilhas, pode explicar a não verificação do pico pré-ovulatório de LH em todos os animais estudados e a menor freqüência de observação deste nesta categoria, no intervalo em que foram colhidas as amostras de sangue.

Dentre os animais em que se observou o pico pré-ovulatório de LH, a amplitude máxima verificada foi, em média, de 4,58 $\pm 0,67$ $\mathrm{ng} / \mathrm{ml}$. Maio et al. (2006) relataram a concentração sérica média de 14,32 ng/ml de LH para o máximo valor observado em novilhas Nelore, com variação de 6,94 a 20,0 $\mathrm{ng} / \mathrm{ml}$. Ambrose et al. (2005) verificaram concentração média de $9,30 \mathrm{ng} / \mathrm{ml}$ no pico de LH de novilhas Holandesas, com valores

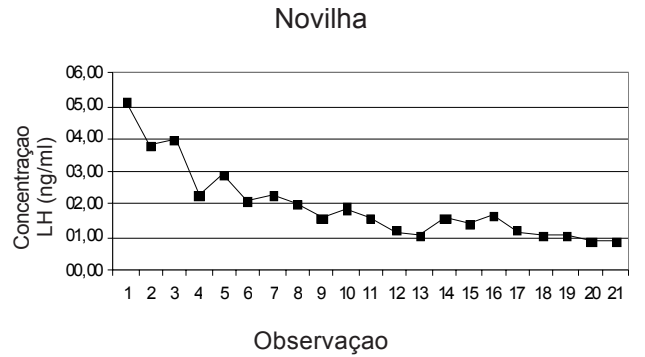

entre 4,60 e $11,80 \mathrm{ng} / \mathrm{ml}$. Provavelmente isto se deva ao estresse do manejo a que animais de criação extensiva foram submetidos no momento das colheitas de sangue, interferindo na amplitude do pico de LH, assim como discutido por Smith and Dobson (2002) devido à ação deletéria dos corticosteróides endógenos, secretados em condições estressantes, sobre o eixo hipotálamo-hipófise-adrenal. Nos animais em que não se observou o pico de LH, foi possível determinar as concentrações médias deste hormônio durante sua liberação pulsátil. Para as novilhas estas foram de $0,90 \pm 0,03 \mathrm{ng} / \mathrm{ml}$ e para as vacas de 0,86 $\pm 0,03 \mathrm{ng} / \mathrm{ml}$. Cupp et al. (1995) relataram valores médios de LH de $0,77 \mathrm{ng} /$ $\mathrm{ml}$ durante o diestro de vacas Bos taurus taurus de corte. Martinez et al. (2005) verificaram, em vacas Hereford tratadas com $17 \beta$ estradiol e dispositivos intravaginais de progesterona, concentrações médias de $0,80 \mathrm{ng} / \mathrm{ml}$ de $\mathrm{LH}$.

\section{CONCLUSÕES}

Sugere-se que nas novilhas o pico préovulatório de $\mathrm{LH}$, provavelmente, tenha ocorrido mais precocemente que nas vacas. São necessários mais estudos sobre o momento do pico de LH em fêmeas bovinas com ovulação sincronizada.

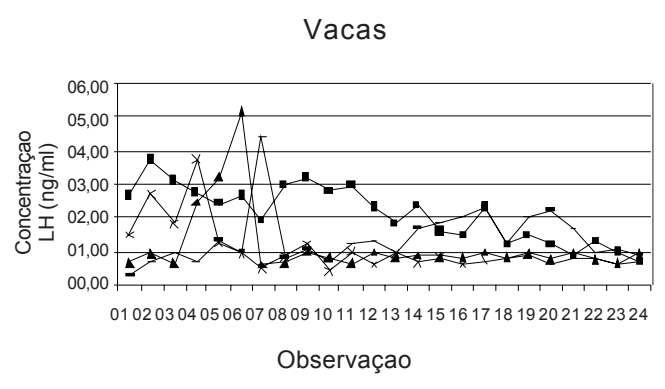

Figura 1. Pico pré-ovulatório de hormônio luteinizante na novilha e nas vacas Nelore submetidas ao protocolo BE-NOR-PGF $2 \alpha-B E$, em observações (coletas de sangue) realizadas a cada 15 minutos, 27 horas após aplicação de 1,0 mg de benzoato de estradiol. (Luteinizing hormone pre-ovulatory peak on Nellore heifer and cows submitted to the protocol BE-NOR-PGF2 $\alpha-B E$, in observations (blood collections) accomplished every 15 minutes, after 27 hours after application of $1,0 \mathrm{mg}$ of estradiol benzoate). 


\section{FERRAZ, OLIVEIRA FILHO, MGAMBARINI, VIU, LOPESE SOUSA}

\section{BIBLIOGRAFIA}

Ambrose, J.D., J.P. Kastelic, R. Rajamahendran, M. Aali e N. Dinn. 2005. Progesterone (CIDR)based timed Al protocols using $\mathrm{GnRH}$, porcine LH or estradiol cypionate for dairy heifers: ovarian and endocrine responses and pregnancy rates. Theriogenology, 64: 1457-1474.

Cupp, A.S., T.T. Stumpf, F.N. Kojima, L.A. Werth, M.W. Wolfe, M.S. Roberson, R.J. Kittok e J.E. Kinder. 1995. Secretion of gonadotrophins change during the luteal phase of the bovine oestrus cycle in the absence of corresponding changes in progesterone or $17 \beta$ estradiol. Anim. Reprod. Sci., 37: 109-119.

Lammoglia, M.A., R.E. Short, S.E. Bellows, R.A. Bellows, M.D. Macneil and H.D. Hafs. 1998 Induced and synchronization estrus in cattle: dose titration of estradiol benzoate in peripuberal heifers and postpartum cows after treatment with an intravaginal progesterone releasing insert and prostaglandinF2 $\alpha$. J. Anim. Sci., 76: 1662-1670.

Maio, J.R.G., G.A.F. Sandoval, E.D.F. Souza, G.P. Nogueira, R.S. Cipriano, F. Perecin e J.M. Garcia. 2006. Concentração sérica de LH em vacas nelore ciclando ou ovariectomizadas submetidas ao tratamento com 2,0 mg de benzoato de estradiol. Acta Scien. Vet., 34 (supl. 1): 360.

Martinez, M.F., J.P. Kastelic, G.A. Bo, M. Caccia and R.J. Mapletoft. 2005. Effects of oestradiol and some of its esters on gonadotrophin release and ovarian follicular dynamics in CIDR-treated beef cattle. Anim. Reprod. Sci., 86: 37-52.

SAS. 2000. Statistical Analysis System. 2000User's guide: Statistic. V. 8. SAS Institute. Cary. 295 p.

Smith, R.F. and H. Dobson. 2002. Hormonal interactions within the hypothalamus and pituitary with respect to stress and reproduction in sheep. Domest. Anim. Endocrin., 23: 75-85.

Archivos de zootecnia vol. 58, núm. 224, p. 760. 